\title{
Topology of magnetic-field-induced current-density field in diatropic monocyclic molecules
}

\author{
Stefano Pelloni, ${ }^{1}$ Francesco Faglioni, ${ }^{1}$ Riccardo Zanasi, ${ }^{2}$ and Paolo Lazzeretti ${ }^{1, *}$ \\ ${ }^{1}$ Dipartimento di Chimica dell'Università degli Studi di Modena, Via Campi 183, 41100 Modena, Italy \\ ${ }^{2}$ Dipartimento di Chimica dell'Università degli Studi di Salerno, via S. Allende, 84081 Baronissi (SA), Italy
}

(Received 30 March 2006; published 17 July 2006)

\begin{abstract}
Concise information on the general features of the quantum-mechanical current density induced in the electrons of a molecule by a spatially uniform, time-independent magnetic field is obtained via a stagnation graph that shows the isolated singularities and the lines at which the current-density vector field vanishes. Stagnation graphs provide compact description of current-density vector fields and help the interpretation of molecular magnetic response, e.g., magnetic susceptibility and nuclear magnetic shielding. The stagnation graph of six cyclic, planar aromatic molecules has been obtained at the Hartree-Fock level via a procedure based on continuous transformation of the origin of the current density formally annihilating the diamagnetic contribution. Some common distinctive elements observed for cyclic aromatic rings $\mathrm{C}_{n} \mathrm{H}_{n}$, with $n$ $=3,4, \ldots, 8$, in the presence of a magnetic field normal to the molecular plane, are discussed. The results can be used for a general discussion of diatropism in aromatic systems.
\end{abstract}

DOI: 10.1103/PhysRevA.74.012506 PACS number(s): 31.10.+z, 31.15.Ar, 33.25.+k, 94.30.Kq

\section{INTRODUCTION}

In his fourth paper on quantization as an eigenvalue problem [1], Schrödinger gave a definition of quantummechanical current density satisfying a continuity equation formally identical to that of classical electrodynamics. A few months later in 1926, Madelung proposed an alternative foundation of quantum theory allowing for a hydrodynamical analogy [2]. Later developments were proposed by Landau [3] and by London [4]. Within the hydrodynamical approach to quantum mechanics, the continuity condition and a vector equation, with the same form as the Hamilton-Jacobi equation of motion of classical mechanics, replace the wave equation [5-11]. The equation of motion can also be recast as Newton's second law, taking into account a nonlocal quantum potential $[5,12]$.

The deep physical significance and the philosophical implications of the proposal put forward by Madelung [2], Landau [3], and London [4] (MLL) can hardly be overemphasized. Bohm showed that the hydrodynamical representation of quantum mechanics offers a broader conceptual framework. It is deterministic and provides an interpretation of physical reality alternative to that of the Copenhagen School $[5,6]$.

Moreover, in a number of instances, the MLL formulation is quite useful in practical terms, e.g., it yields powerful tools for studying molecular magnetic response, which can be rationalized via the electronic current density $\mathbf{J}(\mathbf{r})$ induced by an external magnetic field and by intramolecular magnetic dipoles at the nuclei. The evident advantages of dealing with a vector function of position in real space, instead of a complex wave function depending on $3 n$ space-spin coordinates for an $n$ particle problem, are quite appealing.

The charge density and the current density are called $s u$ bobervables by Hirschfelder [13]. As charge and current density distributions are available, one can ignore the quantum

\footnotetext{
*Corresponding author. Email address: lazzeret@unimo.it
}

mechanical procedure used to get them, and rely on relationships of classical electrodynamics for solving a number of problems quite effectively.

In most cases, a simple representation of the $\mathbf{J}$ field by a set of arrows is sufficient to visualize essential features of systems responding to magnetic perturbations [14-17]. Nonetheless, separate plots of streamlines and modulus of the current-density field are required to understand topological subtleties [18]. The differential Biot-Savart law [19] affords simple and clear interpretations of nuclear magnetic shielding [20-23] and nuclear spin-spin coupling [24-27] via the related concept of property density [28-30].

However, besides providing powerful interpretative tools, maps of current-density field are interesting by themselves for more general reasons. A few problems of physicomathematical interest have received attention. The analysis is carried out by the theory of differential equations and differential topology. Most relevant characteristics are observed in the vicinity of the singularities, also referred to as "equilibrium" and "stagnation" points, i.e., points where the vector field vanishes. The singularities determine the topological structure of the vector field, which is described in compact form by a "stagnation graph" (SG) that conveys essential information [31-33] for understanding magnetic response.

The theory underlying the present study is outlined in the next section. A few observations on magnetic symmetry [34] are recalled in Sec. III. Section IV reports results obtained for a few, neutral or charged, monocyclic conjugated systems described by the general formula $\mathrm{C}_{n} \mathrm{H}_{n}$, customarily classified as "diatropic" [35], which have aroused particular interest in connection with the so-called ring-current model (RCM) [18].

\section{SINGULARITIES AND STAGNATION GRAPH OF A CURRENT-DENSITY FIELD}

The field $\mathbf{J}(\mathbf{r})$ in the neighborhood of a stagnation point at $\mathbf{r}_{0}$ can be described by a truncated Taylor series expansion about $\mathbf{r}_{0}$ as 


$$
\begin{aligned}
J_{\gamma}(\mathbf{r})= & \left(r_{\alpha}-r_{0 \alpha}\right)\left[\nabla_{\alpha} J_{\gamma}\right]_{\mathbf{r}=\mathbf{r}_{0}}+\frac{1}{2}\left(r_{\alpha}-r_{0 \alpha}\right)\left(r_{\beta}-r_{0 \beta}\right) \\
& \times\left[\nabla_{\alpha} \nabla_{\beta} J_{\gamma}\right]_{\mathbf{r}=\mathbf{r}_{0}}+\cdots
\end{aligned}
$$

Standard tensor notation is employed. Summation over repeated greek indices is implied, according to the Einstein convention. The $3 \times 3$ Jacobian matrix $\nabla_{\alpha} J_{\gamma}$, evaluated at the stagnation point $\mathbf{r}_{0}$, has real coefficients. It is nonsymmetric in the absence of molecular point-group symmetry. In the linear approximation [36], only the first term in Eq. (1) is considered and the description of the field about a stagnation point amounts to solving three coupled linear differential equations arranged into a homogenous system. The matrix of the system is the Jacobian matrix.

Reyn [37] reported a paradigm of all possible phase portraits in the vicinity of a stagnation point in threedimensional flow and a classification in terms of the eigenvalues and eigenvectors of the Jacobian matrix. A classification of stagnation points adopting the Euler index (rank, signature) [38] has been proposed [31-33] and widely adopted $[18,39,40]$. The rank $r$ is defined as the number of nonvanishing eigenvalues of the Jacobian matrix, the signature $s$ is the excess of positive over negative eigenvalues, if they are real or pure imaginary [41]. The continuity equation $\nabla_{\alpha} J_{\alpha}=0$ for stationary flow implies that the Jacobian matrix is traceless all over the $\mathbf{J}$ field, and that only two eigenvalues are linearly independent. This places a limit on the possible $(r, s)$. The allowed cases are [31-33,42] as follows:

(a) $(3, \pm 1)$ points corresponding to isolated singularities. Two eigenvalues satisfy the condition $\xi_{3}=-\operatorname{Re}\left(\xi_{1}+\xi_{2}\right)$ (the symbol Re denotes the real part of its argument). If $\xi_{1}$ and $\xi_{2}$ are real (they may also be $\xi_{1}=\xi_{2}$ ), then a node or a saddle point (see Ref. [36] for the nomenclature) is observed in the representation of the flow over the plane of the eigenvectors $\mathbf{t}_{1}$ and $\mathbf{t}_{2}$ corresponding to $\xi_{1}$ and $\xi_{2}$. If $\xi_{1}$ and $\xi_{2}$ are complex conjugate, a focus is found.

(b) $(2,0)$ points; eigenvalues $\xi_{3}=0, \xi_{1}=-\xi_{2}$. For real $\xi_{1,2}= \pm a$ (pure imaginary $\xi_{1,2}= \pm i b$ ), the phase portrait of a saddle (vortex) is observed. The eigenvectors $\mathbf{t}_{1}$ and $\mathbf{t}_{2}$, corresponding to $\xi_{1}$ and $\xi_{2}$, are real in the case of a saddle (they give the direction of the asymptotes through the singularity) and imaginary in the case of a vortex. Saddle- and vortexstagnation lines are continuous manifolds of $(2,0)$ points. Usually these stagnation lines are symmetry determined and lie entirely on the symmetry planes of a molecule. The eigenvector $\mathbf{t}_{3}$ is locally tangent to the stagnation line. $(2,0)$ points can be open lines (this is the case of axial vortices) or form close loops. A toroidal vortex flows around a closed vortex line of $(2,0)$ points [43]. Diamagnetic (paramagnetic) axial vortices of the electronic current density rotate clockwise (anticlockwise) with respect to an observer placed at the north pole of the $\mathbf{B}$ field. The direction of flow about a vortex line is determined by the vorticity, i.e., by the local curl $\boldsymbol{\nabla} \times \mathbf{J}$.

(c) $(0,0)$ (degenerate) points corresponding to three zero eigenvalues of the Jacobian matrix. These loci constitute transition points at which branching of stagnation lines may occur. From the mathematical point of view, the regime variation is due to an exchange between different canonical forms of the Jacobian matrix [18]. Since a $(0,0)$ point corresponds to a transition between pure imaginary and pure real eigenvalues, branching must necessarily occur in correspondence of three zero eigenvalues [31,32].

The denomination "branching point" used for $(0,0)$ singularities is easily understood in practical cases. Consider, for instance, a molecule of $D_{n h}$ symmetry, in the singlet electronic ground state, in the presence of a magnetic field $\mathbf{B}$ along the highest symmetry axis $C_{n}$. In general, in the outer reaches of the molecular domain, the induced electronic current density is diamagnetic, that is, it flows in planes at right angles to $\mathbf{B}$, like the Larmor current that takes place in atoms. In the proximity of the north and south poles, at great distance from the molecular plane, the diamagnetic regime is represented by the primary $(2,0)$ vortex stagnation line parallel to $\mathbf{B}$ along $C_{n}$. Transition to different regimes, e.g., from vortex to saddle flow, or vice versa, takes place closer to the center of charge, i.e., in the regions of higher electron density $\rho(\mathbf{r})$, where the primary vortex line may split into saddle and vortex lines.

Splitting of a stagnation line into several stagnation lines is regulated by a fundamental theorem of topology proved by Gomes $[31-33,42]$ in the form of an index conservation constraint. Let us assume that the index of a saddle (vortex) line is $-1(+1)$. When a stagnation line of index $i_{0}$ splits into $m$ new lines, the sum of the indices of the stagnation lines emerging from the branching point is

$$
\sum_{k=1}^{m} i_{k}=i_{0} .
$$

For instance, a vortex line may bifurcate giving rise to two new vortex lines and one saddle line. This bifurcation process conserves the total index $i_{0}=+1$.

\section{MAGNETIC SYMMETRY OF MONOCYCLIC SYSTEMS}

Molecular point group symmetry, when present, helps determine the overall features of the induced current-density field and of its stagnation graph. $D_{n h}\left(C_{n h}\right)$ magnetic groups $[34,44,45]$ are considered to discuss the symmetry of conjugated cyclic molecules in the presence of a magnetic field perpendicular to the molecular plane. Thus, assuming $\mathbf{B}$ along the $C_{n}$ axis, parallel to the $z$ direction, and indicating by $R$ the time-reversal operator, the magnetic group $D_{n h}\left(C_{n h}\right)$ for, e.g., the cyclopropenium cation, with $n=3$, is

$$
\begin{aligned}
& C_{3 h} \equiv \overline{6} \equiv\left\{\begin{array}{llll}
E & 2 C_{3} & \sigma_{h} & 2 S_{3}
\end{array}\right\}, \\
& D_{3 h} \equiv \overline{6} m 2 \equiv\left\{\begin{array}{llllll}
E & 2 C_{3} & 3 C_{2} & \sigma_{h} & 2 S_{3} & 3 \sigma_{v}
\end{array}\right\}, \\
& D_{3 h}\left(C_{3 h}\right) \equiv \overline{6} \underline{m 2} \equiv\left\{\begin{array}{llll}
E & 2 C_{3} 3 R C_{2} \sigma_{h} 2 S_{3} 3 R \sigma_{v}
\end{array}\right\} ;
\end{aligned}
$$

for $n=4$, e.g., the cyclobutadienyl dication,

$$
C_{4 h} \equiv 4 / m \equiv\left\{E C_{4} C_{2} C_{4}^{3} i S_{4}^{3} \sigma_{h} S_{4}\right\}
$$




$$
D_{4 h} \equiv 4 / m m m \equiv\left\{E 2 C_{4} C_{2} 2 C_{2}^{\prime} 2 C_{2}^{\prime \prime} i 2 S_{4} \sigma_{h} 2 \sigma_{v} 2 \sigma_{d}\right\},
$$

$$
\begin{aligned}
& D_{4 h}\left(C_{4 h}\right) \equiv 4 / m \underline{m m} \equiv\left\{\begin{array}{llll}
E & C_{4} C_{2} C_{4}^{3} 2 R C_{2}^{\prime} 2 R C_{2}^{\prime \prime}
\end{array}\right. \\
& \left.\times i S_{4}^{3} \sigma_{h} S_{4} 2 R \sigma_{v} 2 R \sigma_{d}\right\} \text {, }
\end{aligned}
$$

for $n=5$, e.g., the cyclopentadienyl anion,

$$
\begin{aligned}
& C_{5 h} \equiv\left\{E C_{5} C_{5}^{2} C_{5}^{3} C_{5}^{4} \sigma_{h} S_{5} S_{5}^{7} S_{5}^{3} S_{5}^{9}\right\}, \\
& D_{5 h} \equiv\left\{E 2 C_{5} 2 C_{5}^{2} 5 C_{2} \sigma_{h} 2 S_{5} 2 S_{5}^{3} 5 \sigma_{v}\right\}, \\
& D_{5 h}\left(C_{5 h}\right) \equiv\left\{E C_{5} C_{5}^{2} C_{5}^{3} C_{5}^{4} 5 R C_{2} \sigma_{h} S_{5} S_{5}^{7} S_{5}^{3} S_{5}^{9} 5 R \sigma_{v}\right\},
\end{aligned}
$$

for $n=6$, e.g., the benzene molecule,

$$
\begin{aligned}
& C_{6 h} \equiv 6 / m \equiv\left\{\begin{array}{llllll}
E & 2 C_{6} 2 C_{3} C_{2} & i & 2 S_{3} & 2 S_{6} \sigma_{h}
\end{array}\right\}, \\
& D_{6 h} \equiv 6 / \mathrm{mmm}
\end{aligned}
$$

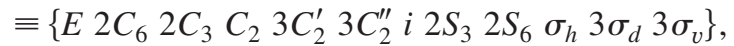

$$
\begin{aligned}
& D_{6 h}\left(C_{6 h}\right) \equiv 6 / m \underline{m m} \equiv\left\{\begin{array}{llll}
E & 2 C_{6} & 2 C_{3} C_{2} & 3 R C_{2}^{\prime} \\
3 R C_{2}^{\prime \prime}
\end{array}\right. \\
& \left.\times i 2 S_{3} 2 S_{6} \sigma_{h} 3 R \sigma_{d} 3 R \sigma_{v}\right\},
\end{aligned}
$$

for $n=7$, e.g., the tropilium cation, $D_{7 h}\left(C_{7 h}\right)$, for $n=8$, e.g., the cyclo-octatetraenyl dication and dianion, $D_{8 h}\left(C_{8 h}\right)$, etc. The essential characteristics of the $\mathbf{J}$ field are directly related to magnetic group symmetry:

(i) A $\sigma_{h}$ plane cannot be crossed by the trajectories.

(ii) $R \sigma_{v}$ and $R \sigma_{d}$ planes can be crossed only by streamlines normal to them in the typical case of vortical regime. If a streamline approaches the $R \sigma$ plane forming an angle different from $\pi / 2$, it is scattered, and a saddle is found. Therefore any (open or closed, vortex or saddle) stagnation line may lie on, but not pass through, an $R \sigma$ plane, and cross perpendicularly, but not lie on, a $\sigma_{h}$ plane. In the present case of $D_{n h}\left(C_{n h}\right)$ symmetries, the stagnation lines are determined by symmetry and are entirely contained in $R \sigma$ planes.

(iii) As the in-plane components of the $\mathbf{J}$ vector vanish all over $R \sigma$ planes by symmetry, the continuity equation for stationary flow $\boldsymbol{\nabla} \cdot \mathbf{J}=0$ is necessarily fulfilled for the perpendicular component, even if $\mathbf{J}$ has been evaluated via approximate quantum mechanical methods.

(iv) The symmetry axes $C_{n}$, parallel to the inducing magnetic field $\mathbf{B}$, lying on $R \sigma_{v}$ planes are necessarily stagnation lines.

\section{THE STAGNATION GRAPH OF $D_{n h}\left(C_{n h}\right)$ SYSTEMS}

A general quantum-mechanical procedure, referred to as continuous transformation of origin of the current densitydiamagnetic zero (CTOCD-DZ) [18,30,46,47], within the damped DZ2 variant $[48,49]$, has been employed at the
Hartree-Fock level of accuracy to obtain the stagnation graph of $D_{n h}\left(C_{n h}\right)$ aromatic cyclic molecules for $n=3,4, \ldots, 8$. The current density evaluated via the CTOCD-DZ and DZ2 procedures is invariant to a change of coordinate system also in the case of approximate calculations [50]. The third-order linear autonomous system of differential equations for the flow was integrated using Runge-Kutta procedures [51].

A preliminary result for $\mathrm{C}_{6} \mathrm{H}_{6}$ had been reported [20]. All the calculations in the present study have been carried out using the high-quality basis sets adopted in this reference. The stagnation graphs obtained for $n=3 \rightarrow 8$ are shown in Fig. 1, which illustrates some general features. As the graphs are quite complicated topological objects in real space, in particular for high $n$ values, a graphic software [52] was developed to obtain three-dimensional representations that can be blown up and rotated by three Euler angles to observe details of the entire SG and of the current-density field in space.

The stagnation graphs of cyclic conjugated hydrocarbons in the presence of a magnetic field perpendicular to the molecular plane are characterized by a number of common features. The diamagnetic flow in the tail regions of the molecular domain is represented by a green open vortex line extending to the boundaries of configuration space. A couple of $(0,0)$ branching points, at the same distance above and below the $\sigma_{h}$ plane, is found for each system. The distance of $(0,0)$ increases from approximately $1.3 \mathrm{bohr}$ for $n=3$ to $2.0 \mathrm{bohr}$ for $n=4$, and to $2.5 \mathrm{bohr}$ for $n=5$. Similar values are found for higher $n$, i.e., $2.5 \mathrm{bohr}$ for $n=6,2.4 \mathrm{bohr}$ for $n=7$, and 2.1 bohr for $n=8$.

Consistent with the Gomes theorem [31-33,42], $n$ diamagnetic vortices, $n$ saddle lines, and a paramagnetic vortex coinciding with the $C_{n}$ principal symmetry axis, originate at the branching point. In Fig. 1, the saddle lines are represented in blue, the diamagnetic (paramagnetic) vortex lines are in green (red). Each diamagnetic vortex crosses the molecular plane in the region close to the midpoint of a C-C bond. Each saddle line reaches the molecular plane in the proximity of the carbon nucleus, just opposite to a C-H bond. The corresponding plots showing streamlines of the current density field in the molecular plane $\left(\sigma_{h}\right)$ are shown in Fig. 2.

Two main remarks need to be made on the basis of these results. First, the central paramagnetic vortex constitutes a leit motiv for the aromatic systems with $D_{n h}\left(C_{n h}\right)$ symmetry. It must exist as required by the Gomes theorem [31-33,42]. Loose statements sometimes reported in the literature, on the assumption that the return current of the diamagnetic C-C bond vortices has opposite direction with respect to the outward flow, may be misleading. The central paramagnetic vortex between two $(0,0)$ points is a physical fact for all aromatic monocyclic molecules. This feature must not be overlooked when aromaticity is discussed in terms of indices like central shielding, or nucleus-independent chemical shift [53].

Second, the assumption frequently made by nuclear magnetic resonance (NMR) spectroscopists that a proton over the ring of carbon atoms in diatropic molecules should undergo a diamagnetic shift caused by $\pi$-electron ring currents [54] needs to be verified in every instance. The maximum modulus of $\pi$ ring currents is observed at the height of $\approx 1$ bohr. If 

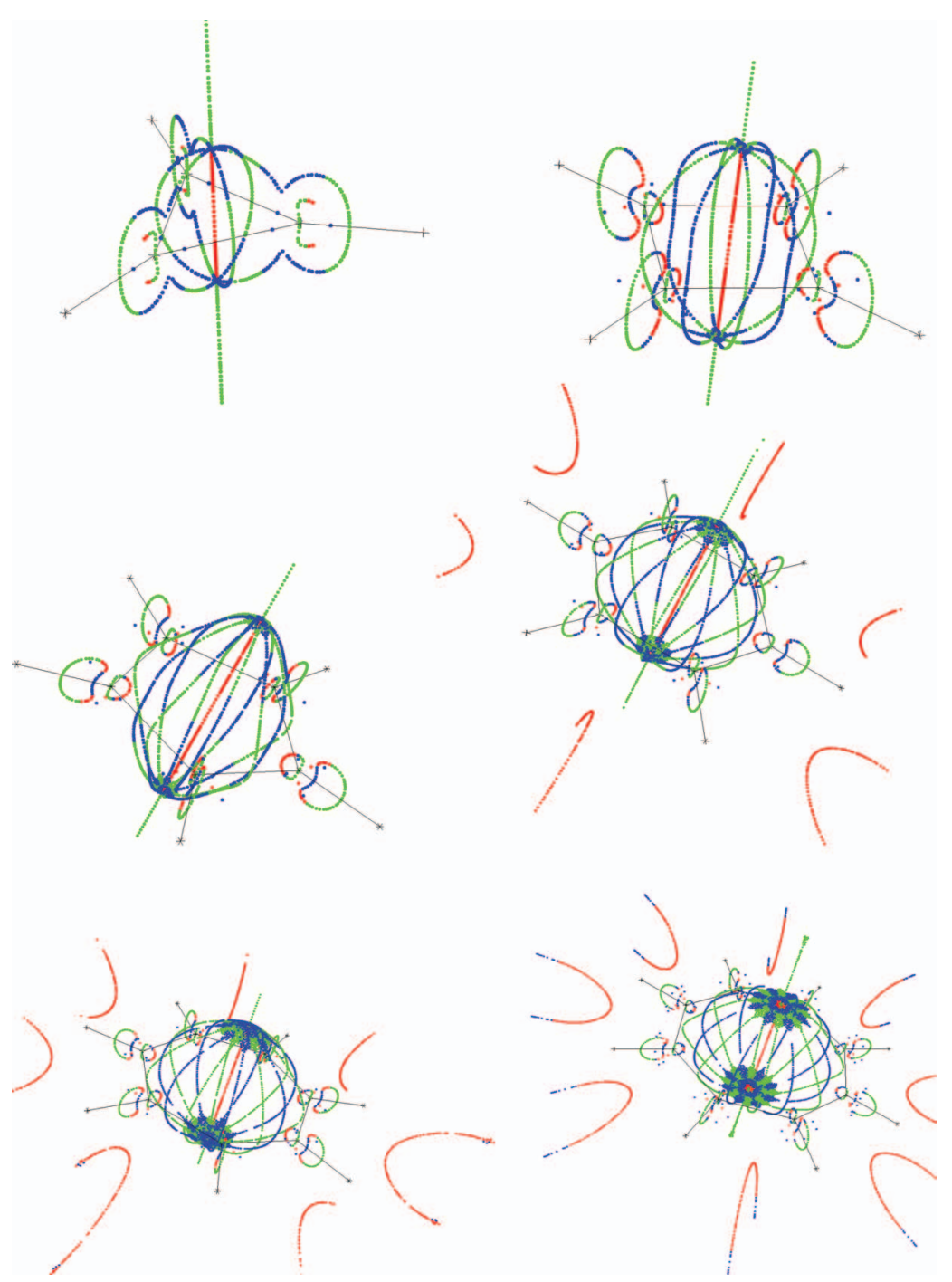

FIG. 1. (Color) The stagnation graph of aromatic cyclic systems $\mathrm{C}_{n} \mathrm{H}_{n}$, for $n=3 \rightarrow 8$. Green (red) indicates diamagnetic (paramagnetic) vortices, and blue saddle lines. Complete graphs and the following Figs. 2 and 3 are available on the web at Ref. [52]. the proton probe is placed over the branching point, the diamagnetic shift may rather be due to the local primary vortex, although the ring current effects fade away at quite long distances [20].

A peculiar pattern characterizes the $\mathrm{SG}$ of $\mathrm{C}_{n} \mathrm{H}_{n}$ aromatic cyclic molecules in Fig. 1. At some distance in between the couple of $(0,0)$ branching points and the molecular plane, the stagnation lines forming the connected part of the SG abruptly change color, denoting a transition from saddle like to diamagnetic vortical regime and vice versa. The points at which the blue (green) lines turn to green (blue) lie all on the same plane $\left(\approx 1.52\right.$ bohr above and below $\sigma_{h}$ in benzene). They are expected to be of $(0,0)$ type. However, no branching was observed for the stagnation lines in the vicinity of these $(0,0)$ singularities.

So far such a behavior, possibly violating the Gomes theorem $[31-33,42,55]$, has neither been predicted nor observed. The theoretical tools available, and the graphic software developed by our group, could not enable us to obtain additional information on the fine structure of the stagnation graph in the vicinity of these loci. Plots of the current density field above and below each $(0,0)$ point are fully consistent with the SG reported in Fig. 1. Further investigations have been planned in the near future to clarify this fact.

Another common feature characterizing the SG of aromatic cyclic molecules for $n>3$ is observed in the region nearby the carbon nuclei. A magnification, see Fig. 3(a) for benzene, reveals the following features. In the upper part of Fig. 3(a), two green vortex lines, belonging to the connected set of seven lines which originate at the $(0,0)$ branching points, and pass close to the midpoint of the $\mathrm{C}-\mathrm{C}$ bonds, are observed. Between them there is a blue saddle line, also originating at the $(0,0)$ singularities. The phase portrait of a saddle is clearly seen on the plot plane, which coincides with that of the molecule, see Fig. 2 and the magnification in Fig. $3(\mathrm{c})$.

Two closed stagnation loops, disconnected from the principal part of the graph lying between the $(0,0)$ points, and independent from one another, are found in the lower part of the figure. Each of them is fully encased in a topological sphere. The streamline pattern observed on the plane, see Figs. 2 and 3(c) contains two juxtaposed diamagnetic vortices, the smaller one with the center on the $\mathrm{C}$ nucleus, the bigger one in the region of the $\mathrm{C}-\mathrm{H}$ bond. Due to incomplete information, such a configuration had been erroneously interpreted in previous works $[18,56,57]$ as evidence for a 

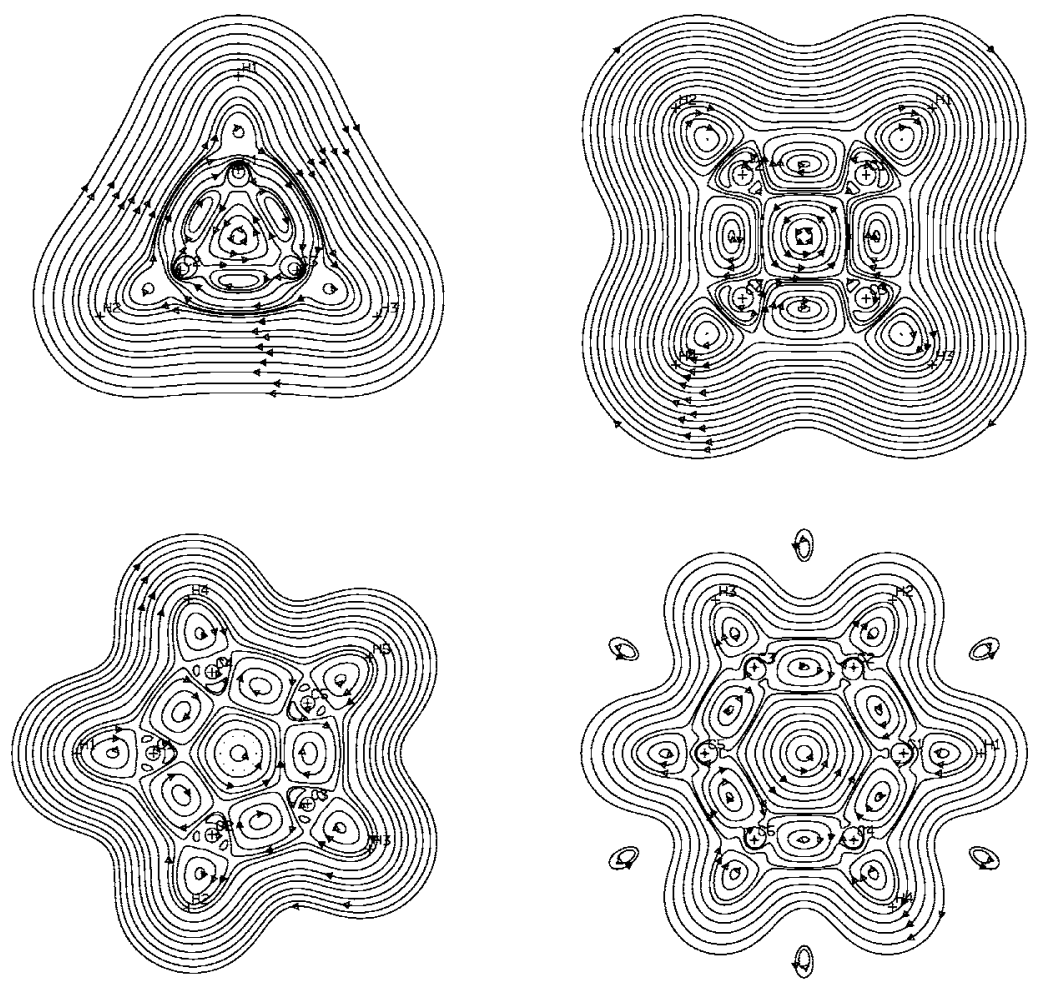

FIG. 2. Streamlines of the current-density vector field induced by a static, uniform magnetic field pointing upward at right angles to the molecular plane of aromatic cyclic systems $\mathrm{C}_{n} \mathrm{H}_{n}$ for $n=3 \rightarrow 8$. Diamagnetic flow is clockwise. The plane depicted contains the nuclei.
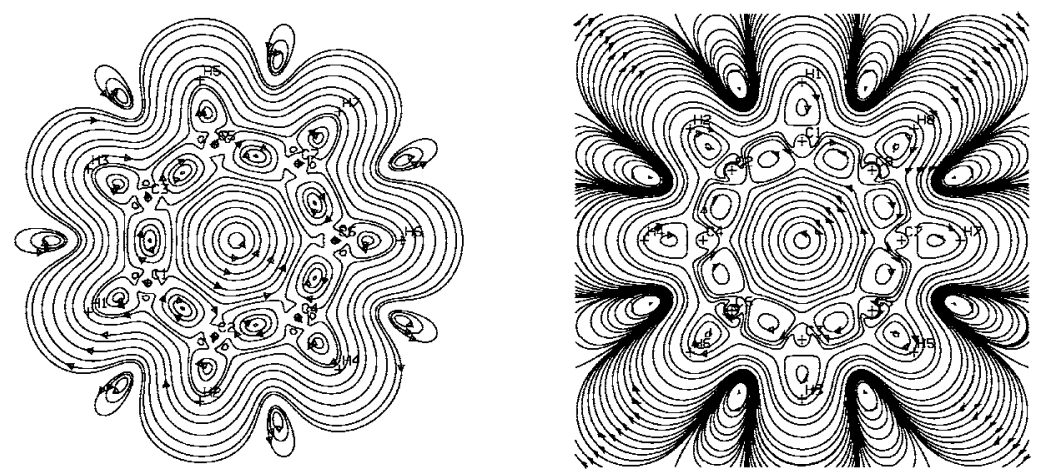

toroidal vortex sectioned by the molecular plane, assuming the existence of only one closed $(2,0)$ vortical manifold.

In fact, both closed stagnation loops are made up of $(2,0)$ vortexlike and saddleline segments, joined at their extremal $(0,0)$ points. The number of saddle (vortex) segments, with topological index $-1(+1)$ within each loop is the same. There are three vortex lines, two red paramagnetic and one green diamagnetic, (two, one red paramagnetic and one green diamagnetic) in the bigger (smaller) loop.

Disconnected subgraphs with these characteristics do not seem to have been predicted by Gomes [31-33,42]. Since the total index after each branching (and within the topological sphere enclosed in each separatrix) vanishes, a subgraph with these characteristics could be referred to as a "fluctuation." of the diamagnetic "vacuum." Its physical meaning might be questioned, unless their stability is proved (from a formal standpoint, such a subgraph can be shrunk to a circumference with vanishing radius and disappear [43]). However, NMR data seem to suggest that the closed stagnation loop through the carbon nucleus is physically plausible. The carbon shielding tensor in benzene is highly anisotropic. Using the intrinsic magnetic dipole of ${ }^{13} \mathrm{C}$ as a probe, the enhanced out-of-plane component, $\sigma_{z z}^{C}$, as large as $190 \mathrm{ppm}$ [58], is attributed to the strong diamagnetic vortex circulating in the electron-rich region nearby the $\mathrm{C}$ nucleus [23]. Although the extension in space of the corresponding stagnation line within the isolated closed loop is quite small, as can be observed in the stagnation graph, the modulus of the electronic current density is very high. Therefore it causes a diamagnetic shift, increasing the shielding of the carbon nucleus to a major extent [23]. Figures 3(a) and 3(c) also show a small paramagnetic whirlpool inside the carbon ring, in the proximity of a carbon nucleus. The corresponding red vortex line belongs to the smaller stagnation loop, vide supra.

Figure 3(b), which contains the set of asymptotic streamlines in the region of a $\mathrm{C}-\mathrm{H}$ bond in benzene, shows peculiar characteristics not described in Fig. 3(c). Two separate figures are given for clearly representing the local flow, due to the complicated structure of the current density field, but the two sets of different trajectories in complementary Figs. 3(c) and 3(b) illustrate inseparable features. However, these perspective views may be insufficient to examine details that are 


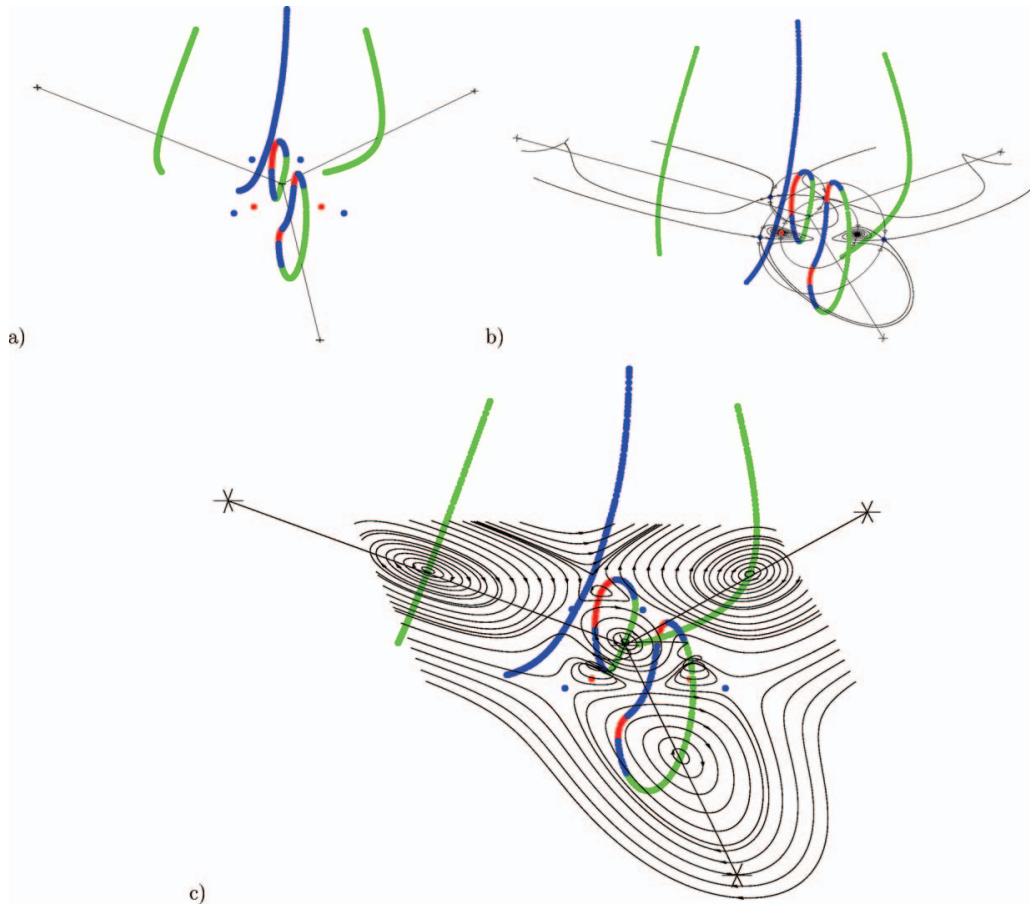

FIG. 3. (Color) (a) Magnification of the stagnation graph of benzene in the region of a $\mathrm{C}-\mathrm{H}$ bond. (b) Selected streamlines in the region of a benzene $\mathrm{C}-\mathrm{H}$ bond. The asymptotic lines connecting each pair of coupled false saddles and coupled foci, $(3, \pm 1)$. The stagnation graph has been superimposed. (c) Magnification of the streamlines in the region of a benzene $\mathrm{C}-\mathrm{H}$ bond. Note the spiral streamlines connecting the false repellor $(3,+1)$ and the false attractor $(3,-1)$. The stagnation graph has been superimposed. better inspected by rotating the local pattern in space via the graphic software available on the web [52].

The plane of the plot, coinciding with that of the molecule, cannot be crossed by any streamline because of the magnetic symmetry of the system, see Sec. III. Trajectories in the vicinity of four isolated $(3, \pm 1)$ singularities outside the carbon ring, namely two red spiral points, i.e., foci, and two blue false saddles visible in the SG in Fig. 3(a), are also observed in Fig. 3(c). In order to show the orbits with nonvanishing out-of-plane component connecting the foci, the flow was integrated by starting at a point in the plane at the distance of 0.01 bohr from that of the molecule.

The two foci, one on each side of the $\mathrm{C}-\mathrm{H}$ bond, are coupled singularities. They look like a source (repellor) and a sink (attractor), respectively, if observed on the molecular plane, see also Figs. 1 and 3(c). In fact, the two foci are connected by trajectories that start spiralling in the proximity of $(3,+1)$, and end up in the region about $(3,-1)$. They are also joined by an asymptotic streamline, leaving $(3,+1)$ and approaching $(3,-1)$, in the direction of the perpendicular eigenvector of the local Jacobian, so that the continuity condition $\nabla_{\alpha} J_{\alpha}=0$ for stationary flow is obeyed, see Fig. 3(b).

Another pair of conjugated $(3, \pm 1)$ points is found outside the benzene ring, at the sides of a $\mathrm{C}-\mathrm{H}$ bond direction [marked in blue in Fig. 3(a)]. For an observer above the molecular plane, each pair of $(3, \pm 1)$ isolated singularities looks like a (false) saddle in Figs. 3(c) and 3(b). However, above and below $\sigma_{h}$, there is a pair of asymptotic streamlines connecting them. These lines leave $(3,+1)$ and approach $(3,-1)$ in the direction of the perpendicular eigenvector of the local Jacobian. These unique streamlines cross perpendicularly the plane containing the stagnation loop through carbon nucleus in two points inside the loop itself. An analogous pattern is found for the pair of $(3, \pm 1)$ points (marked in blue in Fig. 1) inside the benzene ring, at the sides of each $\mathrm{C}-\mathrm{H}$ bond, close to a $\mathrm{C}$ nucleus. The asymptotic streamline connecting the (red) foci lies in between the two pairs of (blue) false saddles.

\section{CONCLUDING REMARKS}

The magnetic response of a molecule to an external magnetic field and to intramolecular magnetic dipole moments at the nuclei can be investigated by analyzing the currentdensity induced in the electron cloud. Quantum mechanics provides powerful tools for evaluating this quantity. In many cases, visualization of electron flow yields simple and valid interpretation for the phenomenology. Moreover, well-known relationships of classical electrodynamics that express induced magnetic moments and magnetic field in terms of a current density field, e.g, the Ampère and Biot-Savart laws, can heuristically be used to evaluate magnetic susceptibilities and magnetic shielding at the nuclei of a molecule.

A precise, rational, and compact description of the current density is obtained by the stagnation graph defined by the singularities of the vector field, i.e., isolated points and onedimensional manifolds that are referred to as vortex and saddle lines. The stagnation graphs of six, neutral and charged $\mathrm{C}_{n} \mathrm{H}_{n}$ aromatic cyclic molecules, in the presence of a static, uniform magnetic field perpendicular to the molecular plane, have been obtained in the present study. The models of current density reported in this paper are characterized by some common properties, e.g, branching out of the primary diamagnetic vortex that flows in the tail regions of the molecular domain into $n$ diamagnetic vortices ending up on the molecular plane. This behavior is observed by the splitting of 
the corresponding primary stagnation line into $n$ saddle and $n$ diamagnetic vortex lines, plus a central paramagnetic vortex line in the direction of the principal symmetry axis $C_{n}$. The presence of a central paramagnetic vortex, which provides a signature of the magnetic response typical of all planar conjugated cyclic molecules, constitutes a quite counter-intuitive aspect. However, this central paramagnetic flow has weak intensity and does not affect the essential diatropicity of aromatics. Another common feature has been observed in the set of streamlines in the vicinity of an aromatic carbon atom. The presence of a strong, localized, diamagnetic vortex around the $\mathrm{C}$ nucleus enhances the out-of-plane component of the magnetic shielding, $\sigma_{\|}$, and is consistent with its large anisotropy.

\section{ACKNOWLEDGMENTS}

Financial support to the present research from the European research and training network "Molecular Properties and Materials (NANOQUANT)," from the Italian MURST (Ministero dell'Università e della Ricerca Scientifica e Tecnologica), via FIRB and PRIN funds, is gratefully acknowledged.
[1] E. Schrödinger, Ann. Phys. 81, 109 (1926), received 21 June 1926. See pages 136ff.

[2] E. Madelung, Z. Phys. 40, 322 (1926), received 25 October 1926.

[3] L. Landau, J. Phys. (USSR) 5, 71 (1941).

[4] F. London, Rev. Mod. Phys. 17, 310 (1945).

[5] D. Bohm, Phys. Rev. 85, 166 (1952).

[6] D. Bohm, Phys. Rev. 85, 180 (1952).

[7] I. Bialynicki-Birula and Z. Bialynicka-Birula, Phys. Rev. D 3, 2410 (1971).

[8] J. O. Hirschfelder and A. C. Christoph, J. Chem. Phys. 61, 5435 (1974).

[9] J. O. Hirschfelder, C. J. Goebel, and L. W. Bruch, J. Chem. Phys. 61, 5456 (1974).

[10] J. O. Hirschfelder and K. T. Tang, J. Chem. Phys. 64, 760 (1976)

[11] J. O. Hirschfelder and K. T. Tang, J. Chem. Phys. 65, 470 (1976).

[12] See Eq. (8a) of Ref. [5]. By combining the first two addenda on the left-hand side of Eq. (2) of Ref. [7], a quantummechanical relationship of the same form as the Newton's second law is obtained for a particle acted upon by the Lorentz force and by the nonlocal Bohm potential, see Eq. (94) of Ref. [18].

[13] J. O. Hirschfelder, J. Chem. Phys. 68, 5151 (1978).

[14] F. Faglioni, A. Ligabue, S. Pelloni, A. Soncini, R. G. Viglione, M. B. Ferraro, R. Zanasi, and P. Lazzeretti, Org. Lett. 7, 3457 (2005)

[15] J. Jusélius, D. Sundholm, and J. Gauss, J. Chem. Phys. 121, 3952 (2004).

[16] Y.-C. Lin, J. Jusélius, D. Sundholm, and J. Gauss, J. Chem. Phys. 122, 214308 (2005).

[17] M. P. Johansson and J. Jusélius, Lett. Org. Chem. 2, 469 (2005).

[18] P. Lazzeretti, in Progress in Nuclear Magnetic Resonance Spectroscopy, edited by J. W. Emsley, J. Feeney, and L. H. Sutcliffe (Elsevier, New York, 2000), Vol. 36, pp. 1-88.

[19] J. D. Jackson, Classical Electrodynamics, 3rd ed. (Wiley, New York, 1999), pp. 175-178.

[20] M. B. Ferraro, P. Lazzeretti, R. G. Viglione, and R. Zanasi, Chem. Phys. Lett. 390, 268 (2004).

[21] S. Pelloni, A. Ligabue, and P. Lazzeretti, Org. Lett. 6, 4451 (2004)
[22] A. Soncini, P. W. Fowler, P. Lazzeretti, and R. Zanasi, Chem. Phys. Lett. 401, 164 (2005).

[23] M. B. Ferraro, F. Faglioni, A. Ligabue, S. Pelloni, and P. Lazzeretti, Magn. Reson. Chem. 43, 316 (2005).

[24] A. Soncini and P. Lazzeretti, J. Chem. Phys. 118, 7165 (2003).

[25] A. Soncini and P. Lazzeretti, J. Chem. Phys. 119, 1343 (2003).

[26] A. Soncini and P. Lazzeretti, Chem. Phys. Lett. 409, 177 (2005).

[27] A. Soncini and P. Lazzeretti, ChemPhysChem 7, 679 (2006).

[28] C. J. Jameson and A. D. Buckingham, J. Phys. Chem. 83, 3366 (1979).

[29] C. J. Jameson and A. D. Buckingham, J. Chem. Phys. 73, 5684 (1980).

[30] P. Lazzeretti, in Handbook of Molecular Physics and Quantum Chemistry, edited by S. Wilson (Wiley, Chischester, 2003), Vol. 3, pt. 1, Chap. 3, pp. 53-145.

[31] J. A. N. F. Gomes, J. Chem. Phys. 78, 4585 (1983).

[32] J. A. N. F. Gomes, Phys. Rev. A 28, 559 (1983).

[33] J. A. N. F. Gomes, J. Mol. Struct.: THEOCHEM 93, 111 (1983).

[34] M. Hamermesh, Group Theory and its Applications to Physical Problems (Addison-Wesley, London, 1972).

[35] From the greek preposition $\delta \iota \alpha$, through, with the idea of penetration, separation, and division in two parts, and the verb $\tau \rho \epsilon \epsilon \epsilon \iota \nu$ (to move in a certain direction, to turn), related to the Akkadian basis "târum", with the same meaning [G. Semerano, Le origini della cultura europea, Vol. II, Dizionario della lingua Greca (Olschki, Firenze, 1994)]. See also the adjective $\delta \iota \alpha ́ \tau \rho o \pi o s$ (changing). In a diatropic system, motion occurs at right angles to the direction $(\tau \rho o ́ \pi o \varsigma)$ of stimulus, e.g., the diamagnetic ring currents induced by a magnetic field B flow in planes perpendicular to it.

[36] E. A. Coddington and N. Levinson, Theory of Ordinary Differential Equations (McGraw-Hill, New York, 1955).

[37] J. W. Reyn, Z. Angew. Math. Phys. 15, 540 (1964).

[38] K. Collard and G. G. Hall, Int. J. Quantum Chem. XII, 623 (1977).

[39] T. A. Keith and R. F. W. Bader, J. Chem. Phys. 99, 3669 (1993).

[40] R. F. W. Bader and T. A. Keith, J. Chem. Phys. 99, 3683 (1993).

[41] If the eigenvalues are complex one defines the signature as the difference between the number of eigenvalues having a posi- 
tive real part and the number of eigenvalues having a negative real part.

[42] J. A. N. F. Gomes, J. Chem. Phys. 78, 3133 (1983).

[43] J. O. Hirschfelder, J. Chem. Phys. 67, 5477 (1977).

[44] B. A. Tavger and V. M. Zaitsev, Sov. Phys. JETP 3, 430 (1956).

[45] C. J. Bradley and B. L. Davies, Rev. Mod. Phys. 40, 359 (1968).

[46] P. Lazzeretti, M. Malagoli, and R. Zanasi, Chem. Phys. Lett. 220, 299 (1994).

[47] S. Coriani, P. Lazzeretti, M. Malagoli, and R. Zanasi, Theor. Chim. Acta 89, 181 (1994).

[48] T. A. Keith and R. F. W. Bader, Chem. Phys. Lett. 210, 223 (1993).

[49] R. Zanasi, J. Chem. Phys. 105, 1460 (1996).

[50] A. Soncini, P. Lazzeretti, and R. Zanasi, Chem. Phys. Lett. 421, 21 (2006).

[51] T. S. Parker and L. O. Chua, Practical Numerical Algorithms for Chaotic Systems (Springer-Verlag, New York, 1986).

[52] The LINUX and Windows versions of the graphic code used to obtain three-dimensional representations of the stagnation graph and current density vector field of a series of molecules can be downloaded at http://theochem.chimfar.unimo.it/ STAGNATION-GRAPH/.

[53] P. von Ragué Schleyer, C. Maerker, A. Dransfeld, H. Jiao, and N. J. R. van Eikema Hommes, J. Am. Chem. Soc. 118, 6317 (1996).

[54] J. A. Pople, W. G. Schneider, and H. J. Bernstein, High Resolution Nuclear Magnetic Resonance (McGraw-Hill, New York, 1959).

[55] At each transition point, the Gomex index changes from +1 $(-1)$ to $-1(+1)$. Thus the theorem is not fulfilled along any given stagnation line. However, the sum of the indices below and above the plane containing all the transition points is the same.

[56] P. Lazzeretti and R. Zanasi, J. Chem. Phys. 77, 3129 (1982).

[57] J. A. N. F. Gomes, Mol. Phys. 47, 1227 (1982).

[58] K. Wiberg, J. D. Hammer, T. A. Keith, and K. Zilm, J. Phys. Chem. A 103, 21 (1999). 